\title{
An unusual cause of superior vena cava obstruction
}

$S^{w}$ uperior vena cava (SVC) obstruction is commonly seen in patients with intrathoracic malignancies. Most cases are due to extrinsic compression by tumour masses. We present the case history of a man with SVC obstruction due instead to an intraluminal tumour which turned out to be adenocarcinoma of an unknown primary.

A 54 year old chronic smoker presented with a 2 week history of progressive facial swelling and dyspnoea. Physical examination revealed signs of SVC obstruction. A CT scan of the thorax showed an expansile tumour mass located within and obstructing the lumen of the SVC lumen (fig 1). Neither CT scanning nor ultrasonography of the abdomen and pelvis showed any evidence of malignancy. Thoracotomy revealed an intracaval tumour obstructing the SVC and invading up to the level of the innominate veins. No primary or secondary tumours could be found. Subtotal tumour resection under extracorporeal circulatory bypass was performed followed by construction of a vascular bypass between the left brachiocephalic vein and right atrium.

Histological examination confirmed adenocarcinoma (fig 2) without any features pointing to a specific primary origin. Immunohistochemical analysis showed positive staining for cytokeratin 7 (CK7) but not for CK20, CEA, thyroid transcription factor-1 (TTF-1), thyroglobulin, and hepatocyte paraffin antibody 1 (HepPar-1), suggesting that the primary tumour might have originated from the lungs or pancreaticobiliary tract. The patient received postoperative radiotherapy to the residual mass followed by platinum based chemotherapy.

While SVC obstruction is not an uncommon presenting feature of certain cancers, ${ }^{12}$ most cases are due to extrinsic compression. Primary or metastatic cancer presenting as an obstructive mass within the SVC is very rare. Reported histology in the literature include thymic carcinoma, primary
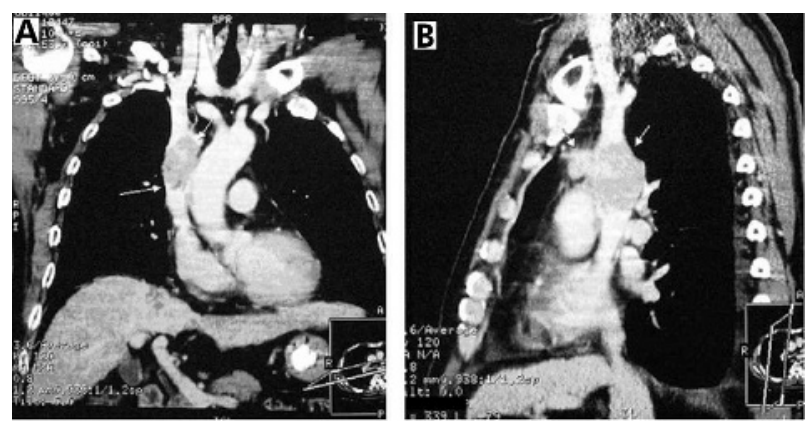

Figure 1 (A) CT image of SVC tumour before surgery showing an expansile mass lying within the lumen of the SVC. (B) CT image of enlarged precaval lymph nodes before surgery.

\section{Learning points}

- Intraluminal tumour is a rare cause of SVC obstruction.

- Establishing a histological diagnosis is challenging because the tumour is inaccessible to percutaneous biopsy. Despite reports of the use of a transvenous catheter for biopsy, thoracotomy remains an important option for obtaining histology and treatment for patients with isolated intraluminal SVC tumour.

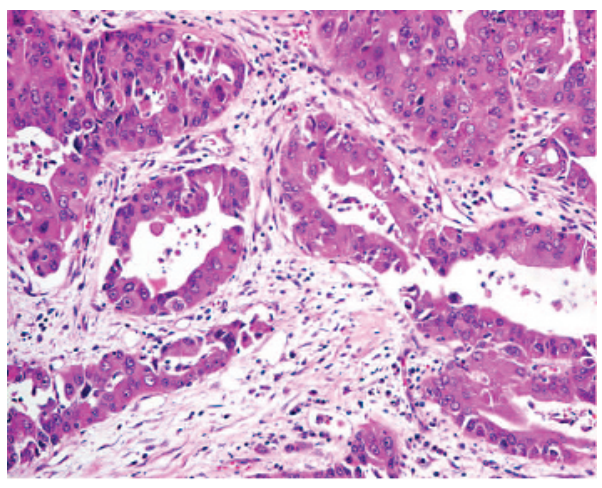

Figure 2 Micrograph showing infiltrative glands composed of moderately pleomorphic cells in desmoplastic stroma. (H\&E; $\times 20)$.

leiomyosarcoma of the SVC, invasive thymoma, paraganglioma, follicular carcinoma of the thyroid, renal cell carcinoma, and lymphoma. Adenocarcinoma of uncertain primary origin presenting initially and solely as an intraluminal SVC tumour is extremely rare.

S L Chan, A A Arifi, A B W Chan, A T C Chan, B B Y Ma Departments of Clinical Oncology, Surgery, and Anatomical, Cellular and Pathology, Chinese University of Hong Kong Prince of Wales Hospital, Shatin, Hong Kong

Correspondence to: Dr B B Y Ma, Department of Clinical Oncology, Chinese University of Hong Kong, Prince of Wales Hospital, 30-32 Ngan Shing Street, Shatin, Hong Kong SAR, China; Brigette@clo.cuhk.edu.hk

\section{REFERENCES}

1 Parish JM, Marschke RF Jr, Dines DE, et al. Etiologic considerations in superior vena cava syndrome. Mayo Clin Proc 1981:56:407-13.

2 Porte $H$, Metois D, Finzi L, et al. Superior vena cava syndrome of malignant origin. Which surgical procedure for which diagnosis? Eur I Cardiothorac Surg 2000;17:384-8. 